that a specific antistreptococcus serum of a high titer applied in both groups of pneumonias-pneumococcus (in double infections) as well as streptococcus-would be the most efficient agent in reducing the present high mortality of these infections. Especially does this apply to the complicating pneumonias-after measles and scarlet fever - as well as the complications following lobar pneumonias.

It may be regarded as an extremely important point in considering the serum treatment of type pneumonias to determine first whether or not the hemolytic streptococcus is coexistent with type pneumococcus.

In the series of necropsies here reported, the hemolytic streptococcus was found to be the immediate cause of death in 77 per cent. of the cases, it having been found in pure culture in serous exudates or the heart's blood in bronchopneumonia and in common with pneumococci in lobar pneumonia cases.

There is no infection more fatal than hemolytic streptococcus septicemia, and this complication as well as the less serious extension of the lung involvement into the pleural and pericardial sacs must be guarded against by well considered therapeutic treatment. If the invasion by the streptococcus can be limited to the lung tissue it is probable that the mortality from the pneumonias, streptococcus and pneumococcus complicated by streplococcus will be markedly reduced.

From a theoretical consideration, it would seem that vaccination would be of practical value in reducing both the case rate and the mortality in type pneumonias and in broncho streptococcus pneumonias, and especially in the pneumonias following measles as well as other complicating processes after measles.

Since vaccination experiments in animals have shown that a high degree of immunity against botk streptococcus and pneumococcus infections can be produced, it would seem reasonable to conclude that a triple vaccine consisting of the hemolytic streptococcus and pneumococci Type I and II would give favorable results.

It may be added that universal vaccination against streptococcus infection should also protect against the complications incident to scarlet fever, as well as against the lung infections of sireptococcus and complicated pneumococcus pneumonias.

Amendments to Sanitary Code in 1917.-The January Bulletin of the New York State Department of Health reviews the amendments to the Sanitary Code which have been adopted during the past year and calls special attention to the following:

Chancroid gonorrhea and syphilis were added to the list of diseases declared to be communicable; it was made obligatory on every physician, when treating a patient affected with any of these diseases to furnish such a patient with a circular of information approved by the State Commissioner of Health and to instruct them as to the precautions to be taken to prevent the communication of the disease to others. It has been made obligatory on every physician to report to his local health officer all persons bitten by suspected rabid animals and all health officer all persons bitten by suspected rabid animals and aca health officers of the occurrence of deaths from communicable diseases other than tuberculosis, it has been made the duty of the local registrar of vital statistics to report immediately to the health officer, the name, age and address of the deceased, whenever a certificate of communicable disease is received, and also the name of the physician who filed the certificate; it is also the duty of the health officer to ascertain whether such person was reported during life as being infected with a communicable disease. A model sanitary code for towns, villages, and third class cities was prepared and recommended for adoption by local authorities. The State Commissioner of Health was given authority to declare extracantonment zones about military camps, and provision has been made for the monthly inspection of such zones by health officers. Regulations were adopted providing for the inspection by health officers of boarding and lodging houses containing people affected with tuberculosis, and licenses are required of boarding houses making a business of boarding children under 12 years of age, away from their parents. More stringent regulations were adopted regulating the practice of midwifery.

\section{A PLEA FOR A MORE FUNDAMENTAL METHOD IN MEDICAL TEACHING}

\author{
ROBERT W. LOVETT, M.D. (BosToN) \\ Major, M. R. C., U. S. Army \\ WASHINGTON, D. C.
}

The recognition of the specialties of medicine made by the Surgeon-General of the United States Army in the early months of the present war represents a step of importance in the progress of medicine, and will obviously promote the efficient care of the wounded. It must to a greater or less extent have an effect on the future of medicine, and it is not unlikely that it may modify medical education, especially as some of the medical schools have in the past few months had experience in the intensive training of groups of military surgeons in the special branches, an experience which in my case has modified extensively my views as to the best methods of teaching.

The experience mentioned has consisted in being associated with four such intensive courses, three at the Harvard Graduate School of Medicine and one at the Army Medical School in Washington. These courses were given to selected military surgeons to acquaint them with some of the fundamentals of orthopedic surgery. To attempt to do more than this in the four, six or eight weeks at our disposal seemed unwise, yet we had in even the shortest of the courses some 130 hours of instruction, far more than any undergraduate would receive in that specialty in any year of his medical course.

Many of these men knew no more orthopedic surgery than is taught in our regular medical school, while others had special experience; but the proposed intensive education naturally had to be adapted for those who knew least. The problem, therefore, was to teach these men as effectively as possible in the time at our disposal. The ordinary method of teaching undergraduates, which consists of instructing the students item by item in the various affections, looked inadequate and proved to be, so that after the first an attempt was made to teach the basic principles of orthopedic surgery and to illustrate these principles clinically.

As the plan apparently proved successful and was intensified in each succeeding course, it has seemed that it might perhaps have a bearing also on the question of undergraduate teaching in this and in other departments in medicine. The matter, therefore, is here discussed from the point of view of a teacher of orthopedic surgery, but with a definite feeling that it has a wider application. I am indebted to my colleague, Capt. Mark H. Rogers, associated with me in the Boston course, for much sympathetic help in the elaboration of the plan.

In the past it has been the custom in general to teach the specialty to the undergraduate by giving didactic or clinical instruction, recitations, or all of them in those different affections which, taken together, might be regarded as constituting orthopedic surgery. Thus scoliosis, clubfoot, the deformities of poliomyelitis, tuberculosis of the spine, flatfoot, torticollis, round shoulders, etc., were taken up one by one, described, demonstrated, illustrated, discussed and dismissed. This is the easy and the obvious way of teaching orthopedic surgery or any allied medical subject, but it is simply making the student, if he 
remembers all that he learns, into a medical encyclopedia with separate, unrelated blocks of information necessarily incomplete in number, because of the impossibility of covering every affection. Although this might pass, and has passed, unchallenged in the undergraduate curriculum, it did not stand the test of intensive instruction when instruction became an unusually serious and responsible business.

The plan was adopted of first taking up general principles, and then illustrating these clinically. It thus became the reverse of the so-called "case system," because the student first heard the principles stated, and after being grounded in them, was made familiar with their application. He did not, as in the "case system," learn to deduce his principles from cinical experience. It was feit that in training necessarily short, but which must be comprehensive, no matter how abundant the supply of clinical material might be, it was safer to ground the student in the fundamentals and teach him to apply them to patients. In this way he would, or should, be better able to approach a new and unusual condition which he had not met clinically.

For the carrying out of this plan it became necessary to arrange the subjects to be taught in groups. The different systems or structures of the body, the joints, the muscles, etc., seemed to offer the best basis of correlation, and in these groups were placed the affections of the different systems. The plan adopted for this method of teaching this specialty was as follows:

\section{AFFECTIONS OF THE JOINTS}

The anatomy of the joints was briefly spoken of, and their general structure, components and varieties described. The physiology of joints was alluded to, and attention called to the facts that use means increase of synovia, that muscles of normal strength are necessary for good joint function and stability, etc. Then came a short statement of the pathologic reaction of joints to trauma, infection or toxins, and it was shown that in the beginning joints react in the same way to the various abnormal influences; that first come hyperemia of the synovial membrane, swelling and increased secretion, and that from this point on one of four cases is generally followed: (1) recovery with a reversai of the acute process; (2) a chronic condition which is a prolongation of the acute; (3) suppuration, and (4) destruction or degeneration, perhaps leaving an ankylosis. Muscle atrophy was shown to be an early accompaniment of all joint affections, and muscle spasm was demonstrated as a reflex tonic contraction of articular muscles in painful and serious joint affections, while ankylosis was shown to be the result of destruction of some part of the joint and to be regarded as a cicatrix.

Cases of any joint affection were then shown, and the students, when possible by personal examination and questioning, were made to identify the general signs described above and to account for them on general pathologic principles. Etiology and special pathologic processes were not at this time discussed. Traumatic affections of joints were then taken up, and a few words said about the peculiarities of different joints in their reaction to trauma; for example, that in the knee the synovial membrane is accessible to examination, but that it is not so in the hip and spine, and that in the latter one must depend more on stiffness, malposition, etc. Cases of traumatic joint affections were then presented, general signs were demonstrated by the students, general conclusions and conclusions as to the pathologic process of the individual case were drawn, differential diagnosis was discussed, and a treatment was formulated to meet the pathologic needs of the individual case. Following this, infections of the joints were taken up and the pathologic peculiarities of tuberculosis described and demonstrated by specimens, lantern slides and roentgenograms. Clinical demonstrations followed along the same lines as those employed with traumatic affections. Acute infections, nontuberculous arthritis, syphilis, intermittent hydrops, hemophilia, etc., were taken up in the same way. Finally, ankylosis was further discussed and clinically demonstrated, positions desirable for stiff joints were elaborated, passive motion and ankylophobia were discussed, and the subject of joints was dismissed.

I had the feeling each time this one department was completed that the student had been furnished with an alphabet by which to spell out the conditions as he met them rather than an incomplete encyclopedia, the items of which he might or might not remember correctly. As this division of the subject has been dwelt on at length, the others may be mentioned much more briefly.

\section{DISEASES ANI) AFFECTIONS OF THE BONES}

The general structure of bone, its growth, regeneration and repair were taken $u p$, and next came a consideration of the various pathologic processes affecting bones, with their peculiarities and characteristics, which were demonstrated clinically and by operations. This included acute and chronic osteomyelitis, syphilis, rickets, osteomalacia, fragilitas ossium, achondroplasia, Paget's disease, etc.

AFFECTIONS OF THE MUSClES OR, MORE PROPERLY, THE NEUROMUSCULAR MECHANISM

The passage of a motor impulse from brain to muscle was described, the upper and lower neurons mentioned, and the distributing function of the synapse showed. The general laws of reflexes were next considered and the general phenomena of muscles discussed, it being pointed out that they are easily fatigued and stretched, always under tension unless paralyzed, and easily developed by exercise. It was then mentioned that the orthopedic surgeon, especially in the field, would have to deal with the deformities caused by focal cord lesions, and lesions of the cerebral motor area and of the peripheral nerves. The essential characteristics of each were pointed out.

The cord lesions were demonstrated, by means of patients, in acute poliomyelitis and fracture of the spine; cerebral lesions, in cerebral spastic paralysis; and peripheral lesions by obstetric paralysis.

\section{STATIC AFFECTIONS}

That muscuiar weakness far short of paralysis might cause deformity was pointed out. The mechanism and anatomy of the foot were described, and its abnormalities under unfavorable conditions of general health, overuse, bad shoeing and overweight were discussed and then clinically analyzed after the general principles had been elaborated. Bad attitude and scoliosis were taken up in the same way, after certain elementary considerations as to the mechanics of the spine and posture in general. 


\section{CONGENITAL DEFORMITIES}

However comprehensive this or any scheme may be, a heterogeneous group of congenital deformities remains to be considered, and these apparently must be taught by the old itemized method after a short statement of our knowledge, or rather lack of knowledge, of the cause and mechanism of congenital deformities.

\section{APPARATUS}

In no part of the subject has the need of generalized instruction been more evident than in the question of apparatus.

This subject has generally been taught through a description by name, in connection with each condition, of some especial apparatus that is used for its treatment, for example, the Thomas splint for hip disease. For the subject of apparatus to be taught effectively, it must be approached in a broader way, and the student made to formulate in each pathologic condition the mechanical requirements, if any, based on the special pathology and his knowledge of joint physiology and mechanics. The student's general attitude is that tuberculosis of the hip requires a brace, the name of which he may or may not recall and of whose mechanical use he knows little or nothing. It is essential to have him learn to recognize that hip tuberculosis requires fixation or traction or both; that this need is determined by the activity and the acuteness of the process in the individual case; that there are many methods of obtaining these ends mechanically, and that the student should recognize the mechanical problem, the crudest way of meeting it, and then the elaboration of crude methods into light and efficient apparatus. This would give the man, especially in the military service, some resource in improvising apparatus, and make him more intelligent in the choice of a method. One would then teach that apparatus was of all kinds: "Wooden splints for fractures are apparatus; so is plaster of Paris. Iron, leather, celluloid, aluminum, papier mâché, etc., also may be. The purposes for which apparatus is used should be shown as being (1) for fixation; (2) for traction; (3) for protection or stilting, and (4) to correct deformity. In short, the student should be tatight to state what he is trying to do in the individual case in mechanical terms, to work out the principle in crude material, and then be made familiar with accepted forms of apparatus suited to such a case.

\section{RECONSTRUCTION}

The question of the rehabilitation of the wounded man and his vocational training must be considered as a special subject, not only because the man disabled in war must be provided for, but also because our knowledge of the rehabilitation of war cripples must be applied later to industrial conditions, and the man crippled in industrial life will never in the future be neglected as he has been in the past.

The general principles on which the best reconstruction work is founded, and the selective aspect and the man's adaptability to a special job as well as the adaptation of the occupation to the individual, are unfolded.

The use of artificial legs and arms, their measurements and fitting, precautions to be taken with the stump and joints of the limb, adaptive additions to artificial limbs, and similar subjects are covered.

\section{SUM MARY}

It will be seen that the crude scheme just described covers all those affections which by the broadest possible interpretation can be included in orthopedic surgery, and that it groups them and gives the student connecting links on which to correlate his impressions, and makes a definite attempt to ground him in fundamentals and to teach him from these fundamentals to elaborate his own details. This method is probably in use in certain departments of medicine and perhaps by some men in all departments; but that it is not the common method would be generally admitted. In the intensive teaching of a special branch to mature men it has been most successful. It would seem as if the undergraduate approaching orthopedic surgery would emerge from his instruction a sounder man if this method were employed than if he merely received instruction in one item after another, however good that instruction might be.

The scheme as presented above is, however, incomplete, because much of the instruction advocated belongs to the departments of anatomy, physiology and pathology, and should be given in those departments and not in orthopedic surgery. However, instruction in most medical schools is imperfectly correlated, and it proved necessary to instruct the men in these special applications of anatomy, physiology and pathology becattse they did not know them well enough to make practical use of them, and the men in question came from many different schools.

A broader scheme, which it is not wise to advocate under war conditions, would point to a more effective correlation of work between the preclinical and the clinical departments in the medical school of the future, without in any way impairing the principle of proceeding from the general to the special as an educational principle.

Smoking and Rifle Shooting.-Some experiments were conducted by the National War Council of the Y. M. C. A. to determine whether smoking affected accuracy in rifle shooting. Smoking and nonsmoking students of the Y. M. C. A. College at Springfield, Mass., participated. In marking the skill of the shooters both the score and the deviation in score covering a certain number of shots before and after smoking were considered. In the first experiment without smoking, five shots were fired, then a rest of thirty minutes and five more shots. In the second, during the interval one cigar was smoked. In a third, two cigars were smoked during an interval of sixty minutes. In a fourth two cigarets were smoked. In the fifth the first experiment was repeated. The shooting was at a target from the prone position with a rifle shooting a 0.22 long cartridge. In the first experiment the group average was 38.1 and after recting, 41 ; before resting the total deviation was 9.23 per cent.; after resting, 7.04 per cent., a decrease in deviation of 12.8 per cent. After one cigar there was a loss of 4.8 in score and an increase in deviation of 24 per cent. After two cigars there was a loss in score of 6 per cent. and an increase in deviation of 26.4 per cent. After two cigarets there was a loss in score of 1.8 per cent. and a decrease in deviation of 1 per cent. In the last experiment, without smoking, but with a rest of thirty minutes, there was a gain of 13.2 in scoring and a decrease of 9.9 per cent. in deviation. The whole group decreased in efficiency after smoking, greater after two cigars than one, but less after the two cigarets than after the cigars. The smokers were affected the least by the smoking, but the nonsmokers were the more efficient after rest. The experiments indicate that soldiers and sportsmen would be better marksmen if they did not smoke. 